\title{
Comparison of Substrate Oxidation during Walking and Running in Normal-Weight and Overweight/Obese Men
}

\author{
Şükrü Serdar Balcı \\ School of Physical Education and Sport, Selçuk University, Alleaddin Keykubat Campus, \\ Selçuklu/Konya, Turkey
}

\section{Key Words}

Indirect calorimetry $\cdot$ Walking $\cdot$ Running $\cdot$ Fat oxidation $\cdot$ Normal weight $\cdot$ Overweight $\cdot$

Obese

\begin{abstract}
Objective: The aim of the present study is to examine the differences in fat and carbohydrate $(\mathrm{CHO})$ oxidation during walking and running between normal-weight and overweight/obese young adult men. Methods: 19 healthy, normal-weight (age $=21.9 \pm 0.7$ years, BMI $=22.6 \pm$ $0.4 \mathrm{~kg}, \mathrm{n}=10$ ) and overweight (age $=21.4 \pm 0.6$ years, BMI $=31.6 \pm 1.1, \mathrm{n}=9$ ) young men volunteered to participate in this study. Body composition was assessed by bioelectrical impedance. Maximal oxygen uptake and maximal fat oxidation rate were determined with indirect calorimetry by using an incremental exercise test on a motor-driven treadmill. The participants' individual preferred transition speeds between walking and running were determined. Indirect calorimetry was used to calculate fat and $\mathrm{CHO}$ oxidation during the resting, walking and running tests. Results: Maximal fat oxidation rates during the graded exercise test were not significantly different between the groups. Changes in $\mathrm{CHO}$ and fat oxidation in the resting, walking and running tests were similar in the normal-weight and overweight groups. Conclusion: The study results suggest that with regard to changes in $\mathrm{CHO}$ and fat oxidation, normal-weight and overweight/obese individuals have similar responses to walking and running at preferred speeds, despite significant differences in oxygen uptake during activity and body composition.




\section{Introduction}

The global increase in the prevalence of overweight and obesity is now reaching epidemic proportions in both developed and developing countries [1]. Regular physical activities, with no changes in diet, are one of the most effective methods of body fat reduction and obesity prevention [2]. Walking and running are the most important forms of physical activity and exercise, and should be encouraged in order to improve public health, given that walking and running are the activities most widely available. Also, they do not require special training or skills [3].

Carbohydrate (CHO) and fat are the most important sources of energy for the body at rest and during physical activity [4]. Walking and running are terms that describe the most preferred movement patterns at lower and higher locomotive speeds, respectively [5]. During walking and/or running activity, the contribution of fat and $\mathrm{CHO}$ oxidation to total energy expenditure is modulated by the intensity of physical activity [6, 7]. The greater the activity intensity, the larger the contribution of $\mathrm{CHO}$ oxidation to the total energy expenditure [8], while fat oxidation interactions with activity intensity and/or type seem to be more complex [9]. In addition, fat oxidation during physical activity may be affected by age [10], gender [7], body composition [11], activity duration [12], diet, and training status [13, 14]. Also, substrate oxidation rates may be different because of different physical activity types [6].

For overweight/obese individuals, the frequency, intensity, and duration of physical activity are more focussed on increasing energy expenditure than on increasing fat oxidation. However, finding the optimal exercise intensity for fat oxidation may improve weight loss and long-term control of body weight [15]. A low resting metabolic rate for a given body composition, a low rate of fat oxidation, and low levels of physical activity are risk factors for body weight gain [16]. Fat oxidation is reduced in obese individuals when measured at the wholebody level or in skeletal muscle at rest $[17,18]$. The reduction in the capacity for lipid oxidation in the skeletal muscle of such individuals may be an integral component of the obese state [19]. It is known that reduced capacity for fat oxidation and inflexibility in regulating fat oxidation in obesity is related to insulin resistance [20], and obese insulin-resistant individuals have been shown to exhibit lower basal fat oxidation than those with normal weight. In the presence of increased adiposity and hyperinsulinemia, reduced fasting fat oxidation develops longitudinally, along with increased fasting CHO oxidation [21].

A number of studies comparing substrate oxidation at different exercise intensities showed that overweight/obese individuals exhibited lower fat oxidation ability and an earlier shift from lipid to CHO-derived fuel than normal-weight or physically active persons [22-25]. On the other hand, several studies have also demonstrated that total fat oxidation during exercise was greater in obese than normal-weight individuals [26-28]. Furthermore, other reports have suggested that fat oxidation during exercise was similar in overweight/ obese and normal-weight individuals [29-32]. However, the effect of body composition on fat oxidation during exercise is unclear because of conflicting results from previous studies [22, 24-32]. Walking and running are the most common types of physical activities prescribed for the prevention and treatment of overweight and obesity. Determining the optimal activity type and intensity for fat oxidation in overweight/obese individuals may help regulating energy balance, increasing weight loss, and preventing weight regain. The purposes of present study, therefore, were to evaluate fat and CHO oxidation rates in normalweight and overweight/obese young men during physical activity and to compare fat oxidation during walking and running at self-selected speeds. The study hypothesizes that the changes in fat and $\mathrm{CHO}$ oxidation during walking and running are different in normalweight and overweight/obese young adult men. 


\begin{tabular}{l|l}
\hline Obes Facts 2012;5:327-338 \\
\hline DOI: $10.1159 / 000339504$ \\
Published online: June 12, 2012 & $\begin{array}{l}\text { C 2012 S. Karger GmbH, Freiburg } \\
\text { www.karger.com/ofa }\end{array}$ \\
\hline
\end{tabular}

Balcı et al.: Comparison of Substrate Oxidation during Walking and Running in Normal-Weight and Overweight/Obese Men

Table 1. Participants' characteristics

\begin{tabular}{lll}
\hline Variables & NW $(\mathrm{n}=10)$ & OW $(\mathrm{n}=9)$ \\
\hline Age, years & $21.9 \pm 0.7$ & $21.4 \pm 0.6$ \\
Height, cm & $175.0 \pm 2.3$ & $176.0 \pm 3.9$ \\
Weight, kg & $69.3 \pm 2.6^{*}$ & $98.3 \pm 5.9$ \\
BMI, kg/m ${ }^{2}$ & $22.6 \pm 0.4^{*}$ & $31.6 \pm 1.1$ \\
Body fat, \% & $13.8 \pm 0.5^{*}$ & $24.2 \pm 1.3$ \\
Lean body mass, kg & $59.7 \pm 2.1^{*}$ & $74.2 \pm 4$ \\
Fat mass, kg & $9.6 \pm 0.6^{*}$ & $24.1 \pm 2.3$ \\
Dry lean mass, kg & $18.5 \pm 1.1^{*}$ & $24.4 \pm 1.3$ \\
Total body water, kg & $41.2 \pm 1.1^{*}$ & $48.9 \pm 2.4$ \\
Waist-to-hip ratio & $0.8 \pm 0.02^{*}$ & $0.9 \pm 0.02$ \\
PTSWR, km/h & $7.4 \pm 0.1^{*}$ & $6.4 \pm 0.2$ \\
VEmax, l/min & $157.1 \pm 11.6$ & $137.1 \pm 6.5$ \\
VO2max, ml/kg/min & $51.8 \pm 1.3^{*}$ & $39.7 \pm 1.6$ \\
pRER & $1.1 \pm 0.02^{*}$ & $1.2 \pm 0.02$ \\
HRmax, bpm & $193.9 \pm 4^{*}$ & $184 \pm 2.4$ \\
REE, kcal/day & $2,040.4 \pm 148.6$ & $2,505.7 \pm 170.4$ \\
\hline
\end{tabular}

$\mathrm{NW}=$ Normal weight; $\mathrm{OW}=$ overweight/obese; PTSWR $=$ preferred transition speed between walking and running; $\mathrm{VE}_{\max }=\max$ imum ventilation; $\mathrm{VO}_{2 \max }=$ maximum oxygen consumption; $\mathrm{pRER}=$ peak respiratory exchange ratio; $\mathrm{HR}_{\max }=$ maximum heart rate; $\mathrm{REE}=$ resting energy expenditure.

* $\mathrm{p}<0.05$; significantly different between normal-weight and overweight/obese subjects.

\section{Participants and Methods}

\section{Participants}

Nine normal-weight and 10 overweight/obese (overweight $n=3$ / obese $n=6$ ) young men participated in the present study (table 1). This study was approved by the Ethics Committee of the School of Physical Education and Sports at Selçuk University. The procedures and risks were thoroughly explained to the participants, and their written and informed consent was obtained. Participants were eligible for participation in the study if they had normal weight (BMI $18.50-24.99 \mathrm{~kg} / \mathrm{m}^{2}$ ) or are overweight/obese (BMI $\geq 25 \mathrm{~kg} / \mathrm{m}^{2}$ ), according to the classification of the World Health Organization [1]. All participants met the following criteria: i) no participation in regular physical activity within the previous year, ii) nonsmokers, and iii) no history of cardiovascular, metabolic, or respiratory diseases.

\section{General Design}

The participants came to the laboratory on 5 separate days for i) anthropometric measurements and the incremental exercise test for the determination of the maximal oxygen uptake $\left(\mathrm{VO}_{2 \max }\right)$; ii) the determination of the individual preferred transition speed between walking and running for the determination of the walking speed and the running speed; iii) a 45-min control test in supine position; iv) a 45-min walking test ( $1 \mathrm{~km} / \mathrm{h}$ slower walk than the individual preferred transition speed); and v) a 45 -min running test $(1 \mathrm{~km} / \mathrm{h}$ faster run than the individual preferred transition speed). Between the tests were at least 3 days but not more than 4 days.

\section{Experimental Design}

Participants were barefoot and in their underwear for the anthropometric measurements. Body weight was measured with a SECA scale (SECA, Hamburg, Germany), and body height with a stadiometer that was incorporated into the scale. Body composition was determined with multifrequency bioimpedance 
(Bodystat QuadScan 4000; Bodystat Ltd., Isle of Man, UK). The accuracy of bioimpedance analysis can be compromised by altered hydration status and extreme obesity [33]. Before testing, individuals were required to keep to the bioimpedance analysis testing guidelines, i.e., not to eat or drink within $5 \mathrm{~h}$ before the test, to maintain normal body hydration, not to consume caffeine and alcohol within $12 \mathrm{~h}$ before the test, not to exercise within $12 \mathrm{~h}$ before the test, not to take diuretics within 7 days before the test, and not to urinate within $30 \mathrm{~min}$. Measurements were performed with the participants lying in a supine position with the knee fully extended in a relaxed state. Two electrodes were placed on the right wrist, one just proximal to the third metacarpophalangeal joints (positive) and one on the wrist next to the ulnar head (negative). Two electrodes were placed on the right ankle with one just proximal to the third metatarsophalangeal joint (positive) and one between medial and lateral malleoli (negative). Multifrequency currents were introduced from the positive leads and travelled throughout the body to the negative leads. Percent of body fat and other body composition parameters were calculated using the manufacturer's software.

All participants were accustomed to walking and running on a treadmill before the beginning of the study (Cosmed S.R.L., T150E, Rome, Italy). A submaximal graded exercise test using the Modified Bruce Treadmill Protocol was conducted while expired gases were collected via facemask and analyzed (Cosmed $\mathrm{K}_{4} \mathrm{~b}^{2}$ portable metabolic system, Cosmed S.R.L., Rome, Italy) for determination of $\mathrm{VO}_{2 \text { max }}$. The test protocol begins at a speed of $2.7 \mathrm{~km} / \mathrm{h}$ at $0 \%$ grade for $3 \mathrm{~min}$ then progresses to $2.7 \mathrm{~km} / \mathrm{h}$ at $5 \%$ grade for $3 \mathrm{~min}$. After this stage, the protocol is identical to that of the Bruce Protocol [34]. The indirect calorimetry system was calibrated prior to each test according to manufacturer specifications. The criteria for achieving $\mathrm{VO}_{2 \max }$ were evaluated as maximum heart rate with respect to age (220 beats/min - age), $\mathrm{VE} / \mathrm{VO}_{2}$ value close to $30 \mathrm{l} / \mathrm{min}$ and respiratory exchange ratio (RER) greater than 1.15 . It was calculated as the average oxygen uptake over the last $60 \mathrm{~s}$ of the test. The physical characteristics of the participants are shown table 1.

Preferred transition speed was determined using much the same protocol as that employed by Rotstein et al. [35]. At the beginning, the treadmill speed was set at a comfortable walking speed of $4 \mathrm{~km} / \mathrm{h}$ for the participant, which was increased by $0.5 \mathrm{~km} / \mathrm{h}$ every minute. The participants were requested to walk as long as they were comfortable and to start running at a particular speed they felt running was more comfortable. At this point, the participant was requested by the test administrator to run for $30 \mathrm{~s}$ and then to walk for $30 \mathrm{~s}$. These walking-running intervals were repeated until the participant was sure with his individual walk-to-run transition speed. If the participant decided to walk, the speed was increased until the participant was certain that running was preferred. After a 15-min rest, a similar procedure was performed by starting the treadmill at a relatively fast speed of $9 \mathrm{~km} / \mathrm{h}$ (speed was set at $8 \mathrm{~km} / \mathrm{h}$ for the overweight/obese) while the participants were running. Speed was then decreased gradually by $0.5 \mathrm{~km} / \mathrm{h}$ every minute until a velocity was reached where the subject preferred to walk. This transition-walking speed was defined as the run-walk transition. The average of the walk-run and run-walk transition speed was defined as the preferred transition speed between walking and running (PTSWR) of the participant.

The participants reported to the laboratory after a 15-hour fast. They were instructed to avoid caffeine, alcohol, strenuous and exhaustive physical activity for 3 days before the experiment. Each participant performed 45 min of walking at $1 \mathrm{~km} / \mathrm{h}$ slower than individual PTSWR, 45 min of running at $1 \mathrm{~km} / \mathrm{h}$ faster than individual PTSWR, and 45-min control test with the participant in supine position. The expired air was measured and analyzed breath-by-breath using an automated online system, and heart rate was monitored and recorded throughout resting, walking and running tests. Each subject was tested at the same time of day (12:00-14:00 a.m.) in order to minimize the effects of diurnal biological variation.

\section{Indirect Calorimetry and Calculations}

Indirect calorimetry was used to estimate $\mathrm{CHO}$ and fat oxidation at rest and during walking and running. The calculations were performed for both the 15-min intervals during the tests and total test times. $\mathrm{CHO}$ and fat oxidation during the tests were calculated according to the stoichiometric equations [36] assuming that the urinary nitrogen excretion rate was negligible:

CHO oxidation $(\mathrm{g} / \mathrm{min})=4.55 \times \mathrm{VCO}_{2}-3.21 \times \mathrm{VO}_{2}$

Fat oxidation $(\mathrm{g} / \mathrm{min})=1.67 \times \mathrm{VO}_{2}-1.67 \times \mathrm{VCO}_{2}$ 


\begin{tabular}{l|l}
\hline Obes Facts 2012;5:327-338 \\
\hline DOI: $10.1159 / 000339504$ \\
Published online: June 12, 2012 & $\begin{array}{l}\text { (c) 2012 S. Karger GmbH, Freiburg } \\
\text { www.karger.com/ofa }\end{array}$ \\
\hline
\end{tabular}

Balcı et al.: Comparison of Substrate Oxidation during Walking and Running in Normal-Weight and Overweight/Obese Men

Table 2. Physiological param-

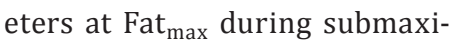
mal graded exercise test

\begin{tabular}{|c|c|c|}
\hline Variables & $N W(n=10)$ & $\mathrm{OW}(\mathrm{n}=9)$ \\
\hline Fat $_{\max }, \mathrm{g} / \mathrm{min}$ & $0.67 \pm 0.01$ & $0.63 \pm 0.02$ \\
\hline Fat $_{\max }, \mathrm{mg} / \mathrm{min} / \mathrm{LBM}$ & $9.85 \pm 0.65$ & $8.5 \pm 0.7$ \\
\hline $\mathrm{VO}_{2} / \mathrm{kg}$ at $\mathrm{Fat}_{\max }, \mathrm{ml} / \mathrm{kg} / \mathrm{min}$ & $21.9 \pm 1.7$ & $21.5 \pm 1.8$ \\
\hline $\mathrm{VO}_{2} \%$ at $\mathrm{Fat}_{\max }, \%$ & $47 \pm 03$ & $54 \pm 04$ \\
\hline RER at Fat $\max$ & $0.78 \pm 0.01$ & $0.81 \pm 0.02$ \\
\hline HR at Fat ${ }_{\text {max }}, \mathrm{bpm}$ & $126.8 \pm 6.2$ & $125.9 \pm 5.7$ \\
\hline
\end{tabular}

NW = Normal weight; OW = overweight/obese; Fat max $=$ maximal fat oxidation rate during submaximal graded exercise test; LBM = lean body mass $(\mathrm{kg}) ; \mathrm{VO}_{2}$ at $\mathrm{Fat}_{\max }=$ oxygen uptake at intensity correspondent at $\mathrm{Fat}_{\max } \% \mathrm{VO}_{2} \max$ at $\mathrm{Fat}_{\max }$ : percentage of maximal oxygen uptake that elicited Fat $t_{\max }$ RER at Fat $\mathrm{T}_{\max }=$ respiratory exchange ratio at intensity correspondent at Fatmax; HR at Fatmax $=$ maximum heart rate at intensity correspondent at Fat max. $_{\text {. }}$

The relative contributions to energy expenditure from $\mathrm{CHO}$ and fat oxidation were calculated using the following equation [37]:

$$
\begin{aligned}
& \% \text { Fat }=((1-\mathrm{RER}) / 0.29) \times 100 \\
& \% \text { CHO }=((\mathrm{RER}-0.71) / 0.29) \times 100
\end{aligned}
$$

Resting energy expenditure was calculated using the equation of Weir [38]. Average values for $\mathrm{VO}_{2}$ and $\mathrm{VCO}_{2}$ were calculated over the last $2 \mathrm{~min}$ for every stage of the modified Bruce treadmill test, and the following variables were identified: highest fat oxidation rate $\left(\mathrm{Fat}_{\max }\right), \% \mathrm{VO}_{2 \max }$ at which the highest fat oxidation was observed, $\mathrm{VO}_{2}$, and RER at the $\mathrm{Fat}_{\max }[6]$.

\section{Statistical Analysis}

Statistical analysis was performed using SPSS for Windows (Chicago, IL, USA). Statistical evaluation of the data was accomplished by using a two- and three-way analysis of variance with repeated-measures design. The two factors were body weight classification (normal weight and overweight/obese) and repeated measures (rest, walking, running). Also, the three factors were body weight classification (normal weight and overweight/obese), test condition (rest, walking, running), and repeated measures (time intervals). When the time effect was significant in the ANOVA of repeated measures, one-way analysis of variance with post-hoc Bonforoni test was applied to identify the tests and/or times responsible for the difference. Unpaired t-tests were used to compare mean values between groups. Statistical significance was set at a $\mathrm{p}<0.05$ level, and data are expressed as mean \pm standard error of the mean.

\section{Results}

The age, height, and maximum ventilation were similar in the normal-weight and the overweight/obese subjects. Body weight, BMI, body fat, fat mass, lean body mass, dry lean mass, total body water, waist-to-hip ratio, preferred transition speed between walking and running, maximal oxygen uptake, peak respiratory exchange ratio, maximum heart rate, and resting energy expenditure were significantly different between normal-weight and overweight/obese groups $(\mathrm{p}<0.05)$.

$\mathrm{Fat}_{\text {max }}$ expressed in lean body mass, oxygen uptake at intensity at $\mathrm{Fat}_{\max }\left(\mathrm{VO}_{2} / \mathrm{kg}\right.$ at Fat $\left._{\text {max }}\right)$, percentage of maximal oxygen uptake that elicited $\mathrm{Fat}_{\max }\left(\mathrm{VO}_{2} \%\right.$ at Fat $\left.\mathrm{Fax}_{\max }\right)$, RER at

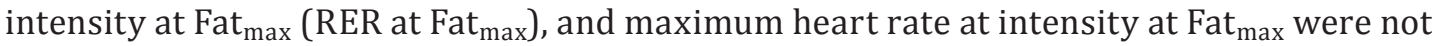
different between the groups ( $\mathrm{p}>0.05)$. 
Table 3. Differences in carbohydrate oxidation, fat oxidation, and energy expenditure between normalweight and overweight/obese participants during resting, walking and running

\begin{tabular}{|c|c|c|c|c|c|c|}
\hline \multirow[t]{2}{*}{ Variables } & \multicolumn{3}{|l|}{ Test } & \multicolumn{3}{|l|}{$\mathrm{F}$} \\
\hline & resting & walking & running & test & group-test & group \\
\hline \multicolumn{7}{|c|}{ CHOox, g/min } \\
\hline NW & $0.21 \pm 0.03 a$ & $0.93 \pm 0.07 b$ & $1.76 \pm 0.16^{c}$ & \multirow{2}{*}{$90.83^{*}$} & \multirow{2}{*}{0.27} & \multirow{2}{*}{0.38} \\
\hline OW & $0.24 \pm 0.04^{a}$ & $0.92 \pm 0.07 b$ & $2.05 \pm 0.25^{c}$ & & & \\
\hline \multicolumn{7}{|c|}{ FATox, g/min } \\
\hline NW & $0.06 \pm 0.01^{\mathrm{a}}$ & $0.38 \pm 0.02 \mathrm{bc}$ & $0.58 \pm 0.07 \mathrm{cb}$ & \multirow{2}{*}{ 79.71* } & \multirow{2}{*}{0.09} & \multirow{2}{*}{1.03} \\
\hline OW & $0.09 \pm 0.02^{\mathrm{a}}$ & $0.40 \pm 0.06^{b c}$ & $0.59 \pm 0.03^{c b}$ & & & \\
\hline \multicolumn{7}{|c|}{$\mathrm{EE}, \mathrm{kcal} / \mathrm{min}$} \\
\hline NW & $1.48 \pm 0.11^{\mathrm{a}}$ & $7.24 \pm 0.40^{b}$ & $12.43 \pm 0.42^{c}$ & \multirow{2}{*}{$297.19 *$} & \multirow{2}{*}{0.67} & \multirow{2}{*}{1.06} \\
\hline OW & $1.78 \pm 0.13^{\mathrm{a}}$ & $7.66 \pm 0.66^{b}$ & $13.60 \pm 1.00^{c}$ & & & \\
\hline
\end{tabular}

NW = Normal weight $(n=10) ; O W=$ overweight/obese $(n=9) ;$ CHOox = carbohydrate oxidation; FATox = fat oxidation; $\mathrm{EE}=$ energy expenditure.

${ }^{*} \mathrm{P}<0.05$; compared between the groups for the tests (two-way repeated measures analysis of variance). Adjustment for multiple comparisons: Bonferroni.

${ }^{a-c}$ Different superscript letters in the same row indicate significant difference with exercise for variables (ANOVA with a Bonferroni post hoc test).

Changes in $\mathrm{CHO}$ oxidation (group-test interaction effect $\mathrm{F}=0.27$, group effect $\mathrm{F}=0.38$ ), fat oxidation (group-test interaction effect $F=0.09$, group effect $F=1.03$ ), and energy expenditure (group-test interaction effect $F=0.67$, group effect $F=1.06$ ), were not different between the groups ( $p>0.05)$. CHO oxidations (test effect $\mathrm{F}=90.83$ ), fat oxidation (test effect $F=79.71$ ), and energy expenditure (test effect $F=297.19$ ) were significantly different among the resting, walking and running tests for both the normal-weight and the overweight/obese groups $(\mathrm{p}<0.05)$. However, according to post hoc analysis, fat oxidation was not different between walking and running for both groups.

Rates of $\mathrm{CHO}$ and fat oxidation were significantly different among the rest, walking and running tests in the normal-weight and the overweight/obese subjects ( $p>0.05)$. CHO and fat oxidation rates during the running test were higher than at rest and during walking. The changes in $\mathrm{CHO}$ (group effect $\mathrm{F}=0.88$, group-condition interaction $\mathrm{F}=0.74 ; \mathrm{p}>0.05$ ) and fat oxidation rates (group effect $F=0.24$, group-condition interaction $F=0.02 ; p>0.05$ ) during the 15-min intervals in rest, walking, and running were not different between the groups. In both the groups, $\mathrm{CHO}$ oxidation rates in the 2 nd and the $3 \mathrm{rd} 15$-min intervals were significantly lower compared to the 1st interval in the walking and running (time effect $\mathrm{F}=$ 16.09, time-condition interaction $\mathrm{F}=9.38 ; \mathrm{p}<0.05$ ). The fat oxidation rates during walking in the 3rd 15-min interval were significantly higher than during the 2nd interval in the normal-weight group. They were significantly higher than the first and 2nd time intervals in overweight/obese group, too. Rates of fat oxidation during running were significantly different between the 3rd and 1st time intervals in the normal-weight group. However, it was significantly different among the time intervals in the overweight/obese group (time effect $\mathrm{F}=49.50$, group-time interaction $\mathrm{F}=3.96$, time-condition interaction $\mathrm{F}=32.67$; $\mathrm{p}<$ 0.05). Oxygen uptake during walking and running in the normal-weight group was significantly higher than in the overweight/obese group in all the time intervals (group effect $\mathrm{F}=$ 72.17, condition effect $F=740.90$, group-condition interaction $F=15.93 ; p<0.05$ ). 
Table 4. Changes in carbohydrate oxidation, fat oxidation, oxygen uptake, respiratory exchange ratio, and heart rate for the normal-weight and overweight participants during resting, walking and running at 0-15, 15-30 and 30-45 minute interval

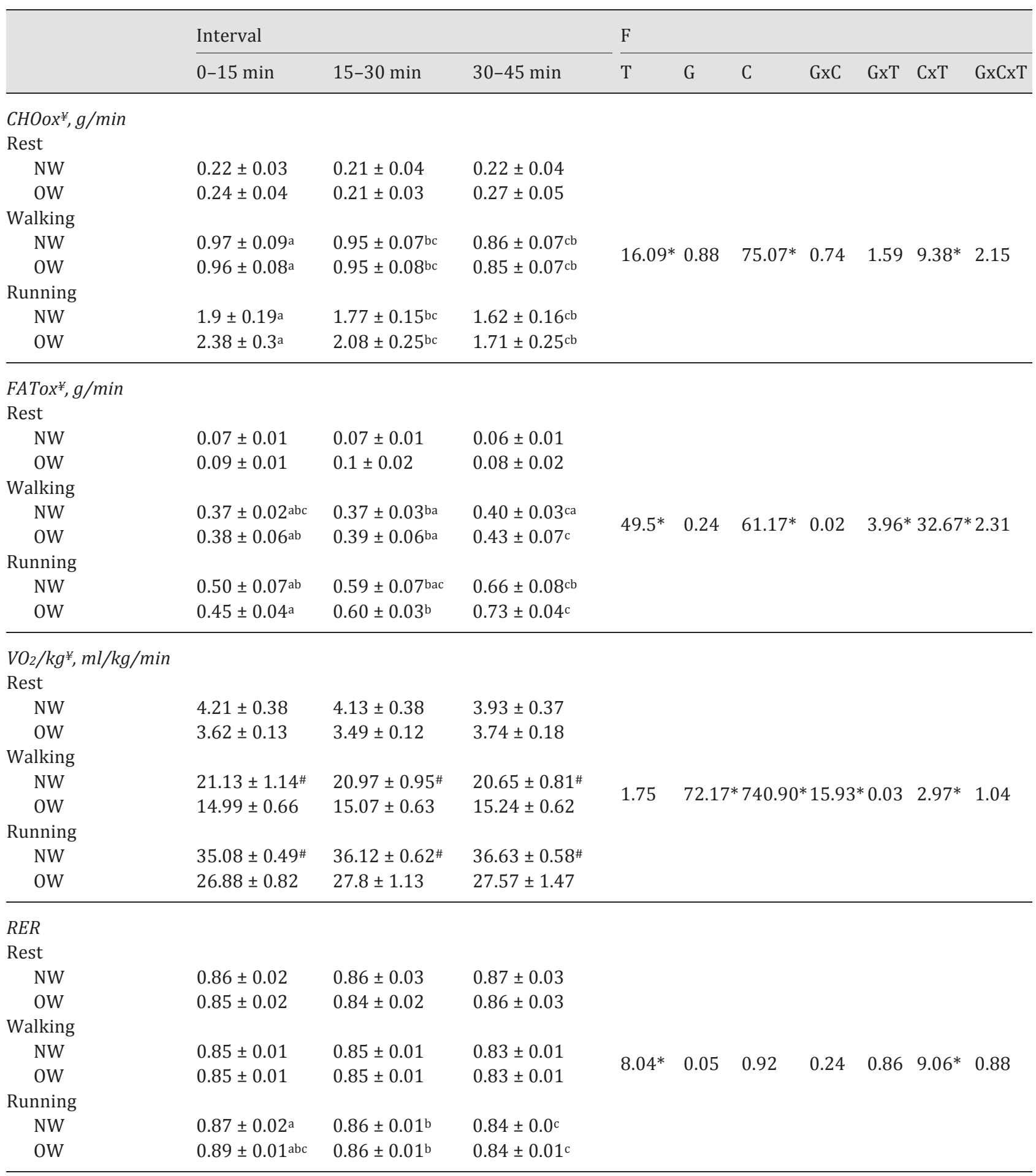


Balcı et al.: Comparison of Substrate Oxidation during Walking and Running in

Normal-Weight and Overweight/Obese Men

Table 4 (continued)

\begin{tabular}{|c|c|c|c|c|c|c|c|c|c|}
\hline & \multicolumn{3}{|l|}{ Interval } & \multicolumn{6}{|l|}{$\mathrm{F}$} \\
\hline & $0-15 \min$ & $15-30 \mathrm{~min}$ & $30-45 \mathrm{~min}$ & $\mathrm{~T}$ & G & $\mathrm{GxC}$ & GxT & $\mathrm{CxT}$ & GxCxT \\
\hline \multicolumn{10}{|c|}{$H R^{\mp}, b p m$} \\
\hline \multicolumn{10}{|c|}{ Rest } \\
\hline NW & $66.4 \pm 3.34$ & $65.2 \pm 3.22$ & $63.6 \pm 3.47$ & & & & & & \\
\hline ow & $63.57 \pm 3.56$ & $62.71 \pm 2.93$ & $63.14 \pm 1.88$ & & & & & & \\
\hline \multicolumn{10}{|l|}{ Walking } \\
\hline NW & $109.14 \pm 3.14$ & $108.71 \pm 2.4$ & $108.29 \pm 1.41$ & & & & & & \\
\hline oW & $106.67 \pm 3.19$ & $104 \pm 2.36$ & $105.22 \pm 2.99$ & 3.15 & 0.07 & $271.51 * 1.51$ & 0.35 & $9.18^{*}$ & 0.17 \\
\hline \multicolumn{10}{|l|}{ Running } \\
\hline NW & $140.29 \pm 4.02^{\mathrm{a}}$ & $146.14 \pm 4.12^{\mathrm{bc}}$ & $149.43 \pm 4.88^{c b}$ & & & & & & \\
\hline ow & $148 \pm 4.52 \mathrm{abc}$ & $153.38 \pm 5.13^{\mathrm{b}}$ & $157.25 \pm 5.34 c$ & & & & & & \\
\hline
\end{tabular}

NW = Normal weight $(n=10) ;$ OW = overweight/obese $(n=9) ;$ CHOox = carbohydrate oxidation; FATox = fat oxidation; $\mathrm{VO}_{2} / \mathrm{kg}=$ oxygen uptake; $\mathrm{RER}=$ respiratory exchange ratio; $\mathrm{HR}=$ heart rate; $\mathrm{T}=$ Time effect $(0-15 \mathrm{~min}, 15-30 \mathrm{~min}, 30-45$ $\min ) ; \mathrm{G}=$ group effect (normal weight, overweight/obese); $\mathrm{C}=$ condition effect (rest, walking, running).

${ }^{*} \mathrm{p}<0.05$; a significant main and/or interaction effect (three-way repeated measures analysis of variance).

${ }^{\#} \mathrm{p}<0.05$; significant differences between normal-weight and overweight/obese groups.

${ }^{¥} \mathrm{p}<0.05$; significant differences among the condition.

${ }^{a-c}$ Different superscript letters in the same row indicate significant difference (one-way analysis ANOVA with a Bonferroni post hoc test).

\section{Discussion}

The purpose of the present study was to examine the change in fat and carbohydrate oxidation rates during walking and running activities, and to determine differences between normal-weight and overweight/obese young adult men. Specifically, the aim was to determine the changes in fat and $\mathrm{CHO}$ oxidation over time (0-45 min). The primary findings of the present study were that, despite significant differences in body composition and aerobic capacity, there were no differences in $\mathrm{CHO}$ and fat oxidation rates when comparing normal-weight and overweight individuals while walking or running. Also, another important finding was that the changes in fat oxidation rates during the time intervals (15 min) in walking and running were similar in the groups.

In the present study, results with regard to resting fat oxidation differences between normal-weight and overweight/obese are consistent with those of the fasting fat oxidation at rest discussed by Blaak et al. [39]. Fasting fat oxidation significantly increased with increasing BMI category $\left(<25,30-35,35-40,>40 \mathrm{~kg} / \mathrm{m}^{2}\right)$, whether unadjusted or adjusted for lean body mass. In addition, Ekelund et al. [40] have emphasized that resting fat oxidation in the obese is significantly greater than in normal-weight young men. The rate of fat oxidation is positively related to body fat mass and negatively related to $\mathrm{VO}_{2 \max }$ [41]. However, this could not be confirmed the present study. The resting fat oxidation rates are similar in normal-weight and overweight/obese young men even after correcting for body weight and lean body mass (data not shown). Similarly, other studies have reported that fat oxidation rates at rest are not affected by body fat and distribution in females [22, 27, 30].

In normal-weight individuals, fat oxidation rates are high over a large range of intensities of physical activity. Besides, at activity intensities higher than Fat ${ }_{\text {max }}$ intensity, fat oxidation rates decreased markedly [42]. Maximal rates of fat oxidation have been shown to be achieved 
at activity intensities between $47-52 \%$ of maximum oxygen consumption in the general

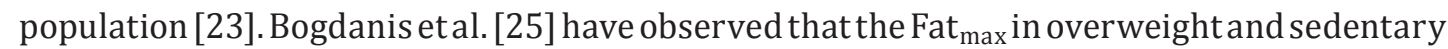
men and women have a low exercise intensity (about $50 \%$ and $40 \% \mathrm{VO}_{2 \text { max }}$, respectively). Ara et al. [43] have reported that young male obese individuals have higher exercise intensity than normal-weight individuals for the Fat $\mathrm{max}_{\text {max }}$ In contrast, the present study results show that $\mathrm{Fat}_{\max }$ and the corresponding exercise intensity are similar in the groups for absolute and relative lean body mass. The interindividual variation in Fat ${ }_{\max }$ remains largely unexplained. Also, body fatness is not a predictor for maximal fat oxidation during exercise [7].

The studies emphasize that overweight individuals need to practise physical activity at a lower intensity than normal-weight individuals [11, 22, 44]. During moderate exercise, overweight men have increased rates of fatty acid oxidation and reduced rates of CHO oxidation when compared with normal-weight men [28]. Previous studies related to the effect of body composition on fat oxidation showed conflicting results. Several studies have found that total fat oxidation during exercise was greater in obese than in normal-weight individuals [26-28]. On the other hand, other reports, comparing substrate oxidation at different exercise intensities, showed that overweight/obese people exhibited lower fat oxidation ability than normal-weight people $[22,24,25]$. However, in the present study, changes in $\mathrm{CHO}$ and fat oxidation rate during the resting, walking, and running tests do not differ between the overweight/obese and normal-weight groups, despite significant differences in oxygen consumption during the walking and running between the groups. Similarly, the studies by Kanaley et al. [29], Ezell et al. [30], Steffan et al. [31], and Mittendorfer et al. [32] have suggested that fat oxidation rates during exercise are similar in obese and lean individuals. Moreover, total fat oxidation in men during exercise is similar for the two obesity phenotypes (visceral fat and abdominal subcutaneous fat) [45]. Also, Geerling et al. [46] have reported that there is no relationship between body fat and fat oxidation during exercise. There are several possible reasons for these conflicting results. One possibility is that overweight/obese individuals may be a heterogeneous group of participants, some of whom may have increased or decreased metabolic efficiency and/or insulin resistance/ sensitivity. The shift in substrate use during exercise may be mediated by insulin resistance rather than by body fat per se [47]. Also, conflicting results can be explained by differences in study designs, such as exercise intensity studied [42] or the age [48] and gender [49] of the subjects. Differences in exercise duration may be especially significant, because the ratio between fat and carbohydrate oxidation during exercise depends on exercise duration $[50,51]$. In the present study, results demonstrate that the relative contribution of fat oxidation to total energy expenditure during walking and running (for $45 \mathrm{~min}$ ) may increase when the duration of activity is increased. Fat oxidation during walking in the 3rd time interval is higher than in the 1 st and the 2 nd time intervals in both groups. Fat oxidation during running in the $3 r$ interval is higher than in the 1st interval in the normal-weight group. However, it is higher than both the 1 st and the 2 nd intervals in the overweight/obese.

Different activity types may affect substrate oxidation during exercise. Fat oxidation is significantly higher during running activity than during cycling at the same relative intensity $[6,14,52]$. Compared to walking, running involves increased velocities, joint range of motion, forces, muscle activity, joint movements, and joint powers [53]. In the present study, CHO oxidations, oxygen uptakes and heart rates were significantly higher during running compared with walking in both groups. Willis et al. [54] have reported that CHO oxidation rate remains very low at speeds less than preferred walking speed $(4.8 \mathrm{~km} / \mathrm{h})$, and fat oxidation provides most of the energy to approach this speed. Maximal fat oxidation occurred at $6.4 \mathrm{~km} / \mathrm{h}$ in normal-weight individuals. At speeds above the preferred walking speed, $\mathrm{CHO}$ oxidation increases and becomes the primary source of energy. A recent study by Entin et al. [55] indicates that fat oxidation rate in normal-weight individuals is not significantly 
affected by walking speed (2.9-6.5 km/h). Anthropometric, kinetic, mechanical, kinematic, perceptual, and particularly energetic factors are likely to play a role in the walk-to-run transition in humans. As speed of locomotion increased to higher than the preferred transition speed, walking, compared with running, became increasingly more dependent upon CHO oxidation [56]. In the present study, fat oxidation during physical activity was not significantly different between walking and running for both groups. However, running activity may lead to slightly greater absolute fat oxidation than walking in both groups. Ganley et al. [56] reported that fat oxidation is higher during running compared with walking at all speeds (between approximately 6.70 and $8.28 \mathrm{~km} / \mathrm{h}$ ) in normal-weight individuals. $\mathrm{CHO}$ oxidation progressively rises across speeds during walking. The lack of increase in fat oxidation with walking suggests that almost all of the extra energy needed to walk at speeds higher than the preferred transition speed is met by increasing CHO oxidation. Running has a greater energy cost than walking on either a track or treadmill [57]. On the other hand, greater fat oxidation may be related mainly to the larger muscle mass involved in running compared with cycling. Muscle mass involved in walking and running activity is similar; however, this may be connected to oxygen uptake levels during physical activity.

The present study has a few limitations. First, fat oxidation during exercise may be influenced by many factors (e.g., age, gender, body composition, activity duration, activity type, diet, and training status). Although several important factors regulating substrate oxidation have been identified, it is apparent that a considerable degree of intersubject variability in substrate utilization persists and cannot be explained by the foregoing factors [58]. Also, insulin resistance was not measured, which is a known factor the balance of substrate oxidation during exercise. Second, energy balance and macronutrient composition of the diet may influence substrate oxidation rates. The participants were informed of dietary measures and content, but they were not monitored during the previous day before the tests. The third limitation is the relatively small sample size, which may have limited the study's statistical power to detect the differences.

\section{Conclusions}

In conclusion, the results of the present study suggest that changes in $\mathrm{CHO}$ and fat oxidation rates during resting, walking, and running are not different between normal-weight and overweight/obese individuals. With regard to changes in $\mathrm{CHO}$ and fat oxidation, normalweight and overweight/obese individuals have similar responses to walking and running at self-selected speeds, despite significant differences in oxygen uptake during activity, maximal oxygen uptake, and body composition. If running speed is slightly faster than the individual preferred transition speed between walking and running (PTSWR $+1 \mathrm{~km} / \mathrm{h}$ ), fat oxidation may be slightly increased in normal-weight and overweight/obese individuals. However, running is not recommended for beginners as it may lead to increased risk of injury.

\section{Acknowledgements}

The author would like to thank Dr. Hamdi Pepe, Dr. Serkan Revan and Deniz Kan for data collection assistance.

\section{Disclosure Statement}

The author has declared that there is no conflict of interest. 
Balcı et al.: Comparison of Substrate Oxidation during Walking and Running in Normal-Weight and Overweight/Obese Men

\section{References}

1 World Health Organization (WHO): Obesity: preventing and managing the global epidemic. Report of a WHO consultation. World Health Organ Tech Rep Ser 2000;894:i-xii, 9, 16-20.

- 2 Slentz CA, Duscha BD, Johnson JL, Ketchum K, Aiken LB, Samsa GP, Houmard JA, Bales CW, Kraus WE: Effects of the amount of exercise on body weight, body composition, and measures of central obesity: STRRIDE - a randomized controlled study. Arch Intern Med 2004;164:31-39.

- 3 Haskell WL, Lee IM, Pate RR, Powell KE, Blair SN, Franklin BA, Macera CA, Heath GW, Thompson PD, Bauman A: Physical activity and public health: updated recommendation for adults from the American College of Sports Medicine and the American Heart Association. Med Sci Sports Exerc 2007;39:1423-1434.

4 Jeukendrup AE: Modulation of carbohydrate and fat utilization by diet, exercise and environment. Biochem Soc Trans 2003;31:1270-1273.

- 5 Tseh W, Bennett J, Caputo JL, Morgan DW: Comparison between preferred and energetically optimal transition speeds in adolescents. Eur J Appl Physiol 2002;88:117-121.

- 6 Achten J, Venables MC, Jeukendrup AE: Fat oxidation rates are higher during running compared with cycling over a wide range of intensities. Metabolism 2003;52:747-752.

7 Venables MC, Achten J, Jeukendrup AE: Determinants of fat oxidation during exercise in healthy men and women: a cross-sectional study. J Appl Physiol 2005;98:160-167.

8 Brooks GA, Mercier J: Balance of carbohyd rate and lipid utilization during exercise: the 'crossover' concept. J Appl Physiol 1994;76:2253-2261.

9 Romijn JA, Coyle EF, Sidossis LS, Gastaldelli A, Horowitz JF, Endert E, Wolfe RR: Regulation of endogenous fat and carbohydrate metabolism in relation to exercise intensity and duration. Am J Physiol 1993; 265:E380-E391.

10 Kostyak JC, Kris-Etherton P, Bagshaw D, DeLany JP, Farrell PA: Relative fat oxidation is higher in children than adults. Nutr J 2007;16;6:19.

-11 Zunquin G, Theunynck D, Sesboüé B, Arhan P, Bouglé D: Comparison of fat oxidation during exercise in lean and obese pubertal boys: clinical implications. Br J Sports Med 2009;43:869-870.

$\checkmark 12$ Pillard F, Moro C, Harant I, Garrigue E, Lafontan M, Berlan M, Crampes F, de Glisezinski I, Rivière D: Lipid oxidation according to intensity and exercise duration in overweight men and women. Obesity (Silver Spring) 2007;15:2256-2262.

13 Bergman BC, Brooks GA: Respiratory gas-exchange ratios during graded exercise in fed and fasted trained and untrained men. J Appl Physiol 1999;86:479-487.

14 Capostagno B, Bosch A: Higher fat oxidation in running than cycling at the same exercise intensities. Int J Sport Nutr Exerc Metab 2010;20:44-55.

15 Jeukendrup AE, Achten J: Fatmax, a new concept to optimize fat oxidation during exercise? Eur J Sports Sci 2001;1:1-5.

-16 Filozof CM, Murúa C, Sanchez MP, Brailovsky C, Perman M, Gonzalez CD, Ravussin E: Low plasma leptin concentration and low rates of fat oxidation in weight-stable post-obese subjects. Obes Res 2000;8:205210.

-17 Kim JY, Hickner RC, Cortright RL, Dohm GL, Houmard JA: Lipid oxidation is reduced in obese human skeletal muscle. Am J Physiol Endocrinol Metab 2000;279:E1039-1044.

18 Consitt LA, Bell JA, Houmard JA: Intramuscular lipid metabolism, insulin action, and obesity. IUBMB Life 2009;61:47-55.

19 Houmard JA: Intramuscular lipid oxidation and obesity. Am J Physiol Regul Integr Comp Physiol 2008; 294:R1111-1116.

-20 Kelley DE, Goodpaster B, Wing RR, Simoneau JA: Skeletal muscle fatty acid metabolism in association with insulin resistance, obesity, and weight loss. Am J Physiol 1999;277:E1130-1141.

21 Poynten AM, Markovic TP, Maclean EL, Furler SM, Freund J, Chisholm DJ, Campbell LV: Fat oxidation, body composition and insulin sensitivity in diabetic and normoglycaemic obese adults 5 years after weight loss. Int J Obes Relat Metab Disord 2003;27:1212-1218.

-22 Pérez-Martin A, Dumortier M, Raynaud E, Brun JF, Fédou C, Bringer J, Mercier J: Balance of substrate oxidation during submaximal exercise in lean and obese people. Diabetes Metab 2001;27:466-474.

-23 Achten J, Jeukendrup AE: Optimizing fat oxidation through exercise and diet. Nutrition 2004;20:716-727.

24 Bircher S, Knechtle B: Relationship between fat oxidation and lactate threshold in athletes and obese women and men. J Sports Sci Med 2004;3:174-181.

25 Bogdanis GC, Vangelakoudi A, Maridaki M: Peak fat oxidation rate during walking in sedentary overweight men and women. J Sports Sci Med 2008; 7:525-531.

26 Horowitz JF, Klein S: Oxidation of nonplasma fatty acids during exercise is increased in women with abdominal obesity. J Appl Physiol 2000;89:2276-2282.

-27 Kanaley JA, Weatherup-Dentes MM, Alvarado CR, Whitehead G: Substrate oxidation during acute exercise and with exercise training in lean and obese women. Eur J Appl Physiol 2001;85:68-73.

28 Goodpaster BH, Wolfe RR, Kelley DE: Effects of obesity on substrate utilization during exercise. Obes Res 2002;10:575-584. 
Balcı et al.: Comparison of Substrate Oxidation during Walking and Running in Normal-Weight and Overweight/Obese Men

29 Kanaley JA, Cryer PE, Jensen MD: Fatty acid kinetic responses to exercise. Effects of obesity, body fat distribution, and energy-restricted diet. J Clin Invest 1993;92:255-261.

-30 Ezell DM, Geiselman PJ, Anderson AM, Dowdy ML, Womble LG, Greenway FL, Zachwieja JJ: Substrate oxidation and availability during acute exercise in non-obese, obese, and post-obese sedentary females. Int J Obes Relat Metab Disord 1999;23:1047-1056.

-31 Steffan HG, Elliott W, Miller WC, Fernhall B: Substrate utilization during submaximal exercise in obese and normal-weight women. Eur J Appl Physiol Occup Physiol 1999;80:233-239.

-32 Mittendorfer B, Fields DA, Klein S: Excess body fat in men decreases plasma fatty acid availability and oxidation during endurance exercise. Am J Physiol Endocrinol Metab 2004;286:E354-E362.

-33 Racette SB, Deusinger SS, Deusinger RH: Obesity: overview of prevalence, etiology, and treatment. Phys Ther 2003;83:276-288.

34 Cooper CB, Storer TW: Exercise Testing and Interpretation: A Practical Approach. New York, Cambridge University Press, 2001, p 242.

-35 Rotstein A, Inbar 0, Berginsky T, Meckel Y: Preferred transition speed between walking and running: effects of training status. Med Sci Sports Exerc 2005;37:1864-1870.

-36 Frayn KN: Calculation of substrate oxidation rates in vivo from gaseous exchange. J Appl Physiol 1983;55: 628-634.

37 Dumortier M, Thöni G, Brun JF, Mercier J: Substrate oxidation during exercise: impact of time interval from the last meal in obese women. Int J Obes (Lond) 2005;29:966-974.

38 Weir JB: New methods for calculating metabolic rate with special reference to protein metabolism. J Physiol 1949;109:1-9.

39 Blaak EE, Hul G, Verdich C, Stich V, Martinez A, Petersen M, Feskens EF, Patel K, Oppert JM, Barbe P, Toubro S, Anderson I, Polak J, Astrup A, Macdonald IA, Langin D, Holst C, Sørensen TI, Saris WH: Fat oxidation before and after a high fat load in the obese insulin-resistant state. J Clin Endocrinol Metab 2006;91:14621469.

40 Ekelund U, Särnblad S, Brage S, Ryberg J, Wareham NJ, Aman J: Does physical activity equally predict gain in fat mass among obese and nonobese young adults? Int J Obes (Lond) 2007;31:65-71.

-41 Kriketos AD, Sharp TA, Seagle HM, Peters JC, Hill JO: Effects of aerobic fitness on fat oxidation and body fatness. Med Sci Sports Exerc 2000;32:805-811.

42 Achten J, Gleeson M, Jeukendrup AE: Determination of the exercise intensity that elicits maximal fat oxidation. Med Sci Sports Exerc 2002;34:92-97.

-43 Ara I, Larsen S, Stallknecht B, Guerra B, Morales-Alamo D, Andersen JL, Ponce-González JG, Guadalupe-Grau A, Galbo H, Calbet JA, Helge JW: Normal mitochondrial function and increased fat oxidation capacity in leg and arm muscles in obese humans. Int J Obes 2011;35, 99-108.

-44 van Aggel-Leijssen DP, Saris WH, Wagenmakers AJ, Senden JM, van Baak MA: Effect of exercise training at different intensities on fat metabolism of obese men. J Appl Physiol. 2002;92:1300-1309.

-45 Numao S, Hayashi Y, Katayama Y, Matsuo T, Tomita T, Ohkawara K, Nakata Y, Tanaka K: Effects of obesity phenotype on fat metabolism in obese men during endurance exercise. Int J Obes (Lond) 2006;30:11891196.

-46 Geerling BJ, Alles MS, Murgatroyd PR, Goldberg GR, Harding M, Prentice AM: Fatness in relation to substrate oxidation during exercise. Int J Obes Relat Metab Disord. 1994;18:453-459.

-47 Braun B, Sharoff C, Chipkin SR, Beaudoin F: Effects of insulin resistance on substrate utilization during exercise in overweight women. J Appl Physiol 2004;97:991-997.

48 Sial S, Coggan AR, Carroll R, Goodwin J, Klein S: Fat and carbohydrate metabolism during exercise in elderly and young subjects. Am J Physiol 1996;271:E983-989.

-49 Chenevière X, Borrani F, Sangsue D, Gojanovic B, Malatesta D: Gender differences in whole-body fat oxidation kinetics during exercise. Appl Physiol Nutr Metab 2011;36:88-95.

50 Ahlborg G, Felig P, Hagenfeldt L, Hendler R, Wahren J: Substrate turnover during prolonged exercise in man. Splanchnic and leg metabolism of glucose, free fatty acids, and amino acids. J Clin Invest 1974;53:10801090.

-51 Watt MJ, Heigenhauser GJ, Dyck DJ, Spriet LL: Intramuscular triacylglycerol, glycogen and acetyl group metabolism during $4 \mathrm{~h}$ of moderate exercise in man. J Physiol 2002;541:969-978.

-52 ChenevièreX, Malatesta D, Gojanovic B, Borrani F: Differences in whole-body fat oxidation kinetics between cycling and running. Eur J Appl Physiol 2010;109:1037-1045.

53 Ounpuu S: The biomechanics of walking and running. Clin Sports Med 1994;13:843-863.

54 Willis WT, Ganley KJ, Herman RM: Fuel oxidation during human walking. Metabolism 2005;54:793-799.

-55 Entin PL, Gest C, Trancik S, Richard Coast J: Fuel oxidation in relation to walking speed: influence of gradient and external load. Eur J Appl Physiol 2010;110:515-521.

56 Ganley KJ, Stock A, Herman RM, Santello M, Willis WT: Fuel oxidation at the walk-to-run-transition in humans. Metabolism 2011;60:609-616.

57 Hall C, Figueroa A, Fernhall B, Kanaley JA: Energy expenditure of walking and running: comparison with prediction equations. Med Sci Sports Exerc 2004;36:2128-2134.

58 Jeukendrup AE, Wallis GA: Measurement of substrate oxidation during exercise by means of gas exchange measurements. Int J Sports Med 2005;26 (suppl 1):S28-37. 